\title{
Variations
}

Variations

Revue internationale de théorie critique

22 | 2019

Gorz, l'intempestif

\section{L'autre sens. Une Théorie critique à la périphérie du capitalisme}

\section{Paulo Arantes en entretien avec Fred Lyra}

\section{(2) OpenEdition}

Journals

Édition électronique

URL : http://journals.openedition.org/variations/1115

DOI : 10.4000/variations. 1115

ISSN : 1968-3960

Éditeur

Les amis de Variations

Référence électronique

Paulo Arantes en entretien avec Fred Lyra, «L'autre sens. Une Théorie critique à la périphérie du capitalisme », Variations [En ligne], 22 | 2019, mis en ligne le 04 mars 2019, consulté le 19 avril 2019. URL : http://journals.openedition.org/variations/1115; DOI : 10.4000/variations.1115

Ce document a été généré automatiquement le 19 avril 2019

Les ami•e•s de Variations 


\title{
L'autre sens. Une Théorie critique à la périphérie du capitalisme
}

\author{
Paulo Arantes en entretien avec Fred Lyra
}

1 Paulo Arantes, né en 1942, est professeur retraité du département de philosophie à l'Université de São Paulo, est un philosophe, essayiste et critique brésilien auquel l'on doit une large production intellectuelle et qui a très activement participé au débat militant-politique du pays, notamment de l'État de São Paulo. Depuis presque vingt ans, il anime le «Séminaire des Mercredis » (Seminário das Quartas), un séminaire pluridisciplinaire et, en un certain sens, non-académique. Considéré comme un «intellectuel destructif», il a publié une douzaine d'ouvrages portant sur plusieurs sujets. Certains, comme Sentimento da Dialetica (1992), Um Departamento françês de ultramar (1994) et O Sentido da Formação (1997, avec Otília B. F. Arantes), dressent le panorama de l'histoire intellectuelle brésilienne. Dans O Fio da Meada (1996) l'histoire intellectuelle traverse tout à la fois la pensée développée au Brésil et celle de France et d'Allemagne. À travers le prisme de l'architecture, et avec Otília B. F. Arantes, il réalise une critique de la pensée du philosophe Jüngen Habermars dans : Un ponto cedo no projeto moderno de Jüngen Habermars (1992). Il a écrit deux ouvrages portant sur la dialectique hegelienne: 0 Ressentimento da Dialetica (1996) et l'ouvrage émanant de sa thèse de doctorat, le seul jusqu'à présent édité en français : L'Ordre du temps. Essai sur le problème du temps chez Hegel, publié en 2000 chez Harmattan. Ses ouvrages les plus récents, Zero à Esquerda (2004), Extinção (2007) et $O$ Novo Tempo do Mundo (2014), rassemblent chacun une collection d'essais critiques du monde contemporain. En outre, il a publié des dizaines d'articles dans des périodiques et revues brésiliens. Nous pouvons citer notamment Tentativa de identificação da Ideologia Francesa ${ }^{1}$ (1990) où, s'inspirant de L'Idéologie Allemande de Marx et Engels, Paulo Arantes essaie de décoder quelle serait la nouvelle idéologie dominante de son temps. Enfin, il dirige actuellement une collection intitulée État de Siège au sein de la maison d'édition Boitempo.

2 Le regard aigu d'Arantes se projette à travers plusieurs angles d'attaque tout à la fois sociaux, économiques, culturels et politiques prenant toujours en compte la dimension nationale et mondiale. La réflexion de la dialectique centre-périphérie est au cœur de sa 
pensée. Autrement dit, le Brésil se transforme en un prisme capable de refléter le monde, comme étant souvent un symptôme privilégié, si nous pouvons parler ainsi, de la crise aiguë que traverse le système capitaliste dans toutes ses dimensions. C'est à travers la prise de conscience de cette matrice de pensée, dont il ressort un autre point de vue, qu'une théorie se construit et peut avoir une voix propre et originale dans le cadre de la reconstruction d'une pensée critique digne de ce nom. Dans cet entretien, parmi d'autres sujets, Paulo Arantes revient notamment sur certains aspects de sa formation au Brésil et en France, sur son vécu des échos de Mai 68 et sa rencontre avec la Théorie Critique (seules deux questions se sont avérées nécessaires pour que le fil de cette pensée se déroule pleinement). Il nous donne notamment quelques pistes, d'une part de ce que serait une Théorie Critique pensée à partir du point de vue d'un pays périphérique et, de l'autre, de ce qu'est une théorie critique qui pense et prend pleinement en compte le temps présent du monde. F.L.

3 Fred Lyra: Vous êtes arrivé à Paris dans l'immédiat post-1968 pour faire une thèse de doctorat sur Hegel sous la direction du professeur Jean-Toussaint Desanti. Nous pourrions commencer alors par cet événement qui fête ses 50 ans en cette année 2018: Mai 68. En outre, on pourrait dire qu'il y avait une convergence intéressante, car au-delà de cet événement politique très important, votre directeur était un Résistant et c'était aussi la période où le structuralisme était hégémonique dans la pensée française et où ce qui allait être connu sous le nom de structuralisme s'affirmait comme la nouvelle vague internationale. Comment revisiteriez-vous aujourd'hui le fait d'avoir vécu in loco les développements immédiats de ce moment politique et théorique singulier?

4 Paulo Arantes : À bien y réfléchir, en fait un croisement mémorable. Comment le revisiteriez-vous 50 ans plus tard? Certainement avec émotion, après tout, ce n'était pas du tout une expérience quelconque, mais surtout avec le sentiment d'être en train de visiter un musée personnel improvisé par un conservateur qui à l'époque ne savait rien, ou presque, des choses que plus tard l'on se verrait dans la contingence de recueillir, et dans un ordre certainement dicté par les pièges de l'anachronisme.

5 Pour être exact, je suis arrivé à Paris en octobre 1969, sans avoir la notion des choses, comme je l'ai dit, à l'exception des généralités archiconnues sur les événements de Mai. Je ne savais pas que je débarquais dans une conjoncture qui allait bientôt être appelée « l'immédiat post-Mai » et dont la signification était disputée par une myriade de petits groupes, une soupe à l'alphabet dans laquelle il n'était pas facile de se reconnaître, à commencer par celle, inhabituelle, de la nébuleuse Maoïste. Il flottait quelque chose comme le sentiment d'un nouveau départ, attesté par la persistance de la rhétorique insurrectionnelle, alimentée du reste par la campagne répressive du Ministre de l'Intérieur.

6 Pour un Brésilien nouveau venu, cette sensation de reprise sur le chemin n'a pas cessé de faire sens, dans une certaine mesure en ce moment on respirait le même air de famille au centre et à la périphérie. Voyez si l'analogie ne fonctionne pas. Dans ce même mois d'octobre 1969, déjà installé à Paris et très loin de la Dictature, le critique littéraire brésilien Roberto Schwarz commença à mettre sur le papier un premier bilan du mouvement politico-culturel qui au Brésil avait répondu au coup d'État de 1964 par une floraison de l'opposition intellectuelle jamais vue, hormis paradoxalement, parce que le régime était oppressif et tendait vers la terreur qui arriverait bientôt dans toute force à partir du AI-5. Et c'est ainsi que vint l'heure de la conversion intellectuelle au militantisme insurrectionnel, dont l'ethos guerrier et volontariste rayonnant de La Havane se nourrissait et se justifiait, par la conviction que la société contenue par la force 
restait égale à elle-même et agitée comme toujours, au point d'ébullition que l'avait surprise la contre-vapeur de la Guerre Froide. Une cocotte-minute à partir de laquelle il suffisait d'enlever le couvercle. Nous savons que, contrairement à l'Italie, où, durant le même automne chaud, la stratégie de tension faisait ses premiers pas, en France la frontière de la lutte armée n'a jamais été franchie. Pourtant, durant ces derniers mois de 69, le fantôme de Mai fut appelé à gauche et à droite pour annoncer, ou faire peur, quelque chose comme une guerre civile en route, en brassant dans le même chaudron qui avait déjà débordé en 68 . Et cela rappelait beaucoup de nos préparatifs de révolution en Amérique latine, les grandes attentes qui ont été rassemblées dans notre " soixante-huit » très particulier de défi à une Dictature encore honteuse, comme il a été dit plus tard.

7 Car la conjoncture française que je devais observer in loco, dans la condition très limitée d'un doctorant à plein temps, de 1969 à 1973, a connu ainsi une montée similaire, par exemple, dans l'hégémonie idéologique des groupes d'extrême gauche engagés à transformer la répétition générale de Mai en une affiche permanente. Quand je parle d'hégémonie, je pense à Sartre et Foucault dans la rue vendant la Cause du Peuple ${ }^{2}$. Et la date limite de 1973, parce que précisément durant la semaine de juin de cette année où j'ai défendu ma thèse, le Ministre Marcelin a dissout la Ligue communiste. Le mois suivant, j'étais de retour au Brésil sans savoir avec certitude ce qu'après tout j'avais vécu.

Vécu, par ailleurs, est une façon de dire. J'ai à peine suivi à distance, par la presse et les magazines interposés. Aussi incroyable que cela puisse paraître, je n'ai mis les pieds à Nanterre, point zéro du tremblement à venir, que deux fois, pour m'inscrire et, des années plus tard, pour la soutenance de la thèse. Dans les premiers temps, je ne me suis éloigné de ma table de travail que pour quelques consultations à la Bibliothèque Nationale. Après, même pas ça. Je n'ai pas non plus visité le parc thématique dans lequel Vincennes s'était transformé. Le spectacle de la pensée 68 se résumait à quelques cours de Foucault au Collège de France, et c'est tout. J'ai circulé très peu ou presque pas en dehors du cercle d'amis brésiliens plus ou moins dans les mêmes conditions de température et pression académiques. Aux alentours néanmoins le temps brûlait. À premier vue ça devrait donner l'impression d'étrangeté, d'être au bord de l'aliénation. En y regardant bien, c'était ni plus ni moins que si j'avais été en train d'écrire ma thèse à São Paulo, à part la culture de la ville. C'est que, à la rigueur, je me sentais entièrement chez moi, car le Hegel que j'étais assidûment en train de lire, annoter et expliquer, était ni plus ni moins, un personnage français. A commencer par le genre historiographique en question, lecture structurelle à côté du texte, suivant les préceptes de la plus traditionnelle école française, actualisée par Gueroult et Goldshmidt. L'intention matérialiste n'y changeait pas grand-chose, et, le marxisme étant professé dans la maison du genre «occidental », doublait d'abord le pari dans la perception que l'essentiel d'une philosophie résidait dans une certaine structure, dans la façon dont un certain contenu de l'expérience se sédimentait. De plus, un Hegel dont on était immanquablement initié à la lecture par le commentaire de Jean Hyppolite de la Phénoménologie de l'Esprit, qui à son tour répercutait toujours les effets collatéraux du grain planté dans le cours inaugural de Kojève dans les années 30 du siècle dernier. Il n'est pas question maintenant de les répertorier, il suffit de rappeler que les traces de ce mémorable Hegel, à bien dire inventé de toute pièce, peuvent être reconnues dans la dialectique de la reconnaissance de l'Être et le Néant, ou dans les élucubrations de Lacan sur le stade du miroir. «Mon » Hegel prenait une autre route, mais le tableau des références, en faveur ou contre, était d'un bout à l'autre français. Sans 
parler de la bizarrerie d'étudier Hegel en France. Dans une bonne logique, pour rester chez soi, même hors-de-soi, ainsi simple.

Ou d'un autre côté, pas si simple, car il serait nécessaire de reconstituer l'ingénierie culturelle particulière qui a permis la construction d'un Département de Philosophie française dans une périphérie éloignée d'outre-mer : de plus, qu'une telle greffe ait non seulement triomphé et pris une direction disons « progressiste » et bien dans la direction d'une importante tradition critique brésilienne, qualifiée par certains de "modeste radicalisme de classe moyenne ", d'ailleurs pas du tout modeste, étant donné le sombre conservatisme local qui est finalement tombé sur le pays avec une force destructrice totale en 1964. Un transfert de techniques intellectuelles et pas une inculcation de doctrines, du reste relativisées dans leur valeur nominale par l'emploi bien calibré de ces mêmes « technologies » d'étude et d'interprétation. Nous nous voyions donc comme des professionnels, au point de considérer, par exemple, l'existentialisme ambiant, alors en vogue, comme une chose d'amateur. Tout bien pesé, un cas d'aliénation productive, une combinaison plus qu'improbable de colonisation mentale et d'illuminisme. Ainsi, quand au début des années 1960, le structuralisme atterrit avec toute sa force, il a été sobrement reçu comme un vieil ami de la maison, dans lequel on reconnaissait une sorte de revanche de la philosophie universitaire française de l'époque de Brunschvig et consorts contre la vague phénoménologique de l'immédiat après-guerre et tout son cortège de lettres majuscules - Conscience, Existence et ainsi de suite marchant vers le concret. Une adhésion au structuralisme serait ainsi redondante, presque absurde, au-delà de faire l'économie des extravagances inhérentes à ces rebondissements spectaculaires. On lisait le manifeste anti-Sartre du dernier chapitre de la Pensée Sauvage à travers le filtre du souvenir de LéviStrauss enseignant à São Paulo à la fin des années trente. D'où aussi l'encadrement d'Althusser: nous nous y sommes opposés, moins pour ne pas embarquer dans le faux dilemme marxisme/structuralisme que parce qu'on le considérait comme un énorme malentendu, nourri par la bonne et vieille épistémologie française du Concept, tournant autour de la distinction, si kantienne et universitaire, entre objet réel et objet de connaissance.

Les choses en étaient là quand Mai est arrivé. Au Brésil aussi, mais confiné au monde culturel-étudiant déjà mobilisé contre la Dictature. En regardant à distance la scène philosophique française, on voyait avant tout un drame familial. Dans un premier acte la revanche de Sartre, le suivant un Foucault rafraîchi, en avant un nouveau tournant et Lacan déclarait que les structures étaient descendues dans la rue, mais que ce n'était pour ça que la descendance maoïste d'Althusser cessait de s'insurger contre la leçon du maître, et ainsi de suite. Mais de la péripétie en effervescence le panorama que l'on commençait à entrevoir dans le sillage de Mai quand je suis arrivé à Paris était caractéristique d'un paradoxal vol de nouveaux mandarins idéologues de la contestation, du retour du structuralisme vêtu d'un nouvel habit de gauchisme, d'une nouvelle hégémonie enfin autour du concept-roi de Discours, transgressif de préférence. En un mot, qu'évidemment je ne peux pas m'expliquer minutieusement, prenait corps quelque chose comme une alternative imaginaire de subversion globale que, quelques années plus tard, Castoriadis baptiserait Idéologie Française, et qui serait exporté vers les Etats Unis sous la dénomination de French Theory.

11 Au milieu de ce tourbillon, que peut-on attendre d'un simple étudiant brésilien des aventures françaises de la dialectique hégélienne? Face à l'énormité de la tâche - réagir à son époque à travers un doctorat, genre par définition inepte -, il n'avait qu'à se 
conformer à ce qui était en fait prescrit par son certificat de naissance : sous la surface d'un argument matérialiste classique - la centralité du travail dans la redéfinition hégélienne du temps - un sous-texte où il ne serait pas trop difficile de reconnaître un règlement de comptes avec le dernier tournant de la pensée soixante-huitarde autour de cet épouvantail que tout le monde aimait déchaîner. Je fais référence au livre dans lequel Gérard Lebrun montrait justement que la fameuse dialectique n'était rien d'autre qu'une façon de parler, un "discours", toutefois extrêmement efficace dans sa fonction de démolition, une étonnante ruse diversioniste, d'ailleurs de tous les deux, le philosophe et son interprète 3 . Publiée en 1972, La patience du concept a été malheureusement occultée par la publication capitale du Manifeste de l'immédiat post-68, l'Anti-〔Edipe. C'étaient les désirants qui entraient en scène en transformant la rupture qui n'a pas eu lieu dans la célébration pop. Mais, j'étais alors déjà sur le dernier tronçon et j'ai laissé pour le retour la tentative d'identification de ce nouveau jargon de l'authenticité soixante-huitarde, sur lequel seulement beaucoup plus tard j'ai écrit une étude, Tentativa de identificação da Ideologia Francesa, à laquelle vous vous référez, pour être plus exact, en 1990, la conjoncture mondiale étant d'ailleurs complètement différente. La lutte pour l'hégémonie dans les appareils culturels a été transférée vers les campus américains, où prospérait un cartel mêlant essentiellement trois consortiums : la French Theory, le doux cosmopolitisme éthique de la Théorie de l'Action Communicative et les mutants nordaméricains du tournant culturel post-analytique, ayant en commun la gravitation de l'ensemble autour de l'omniprésent paradigme du langage, qui avait repoussé le précédent, dit de la Production ou Travail, et sa partenaire d'anciens combats, la Conscience. Encore des éclats de l'explosion de Mai, que personne ne connaissait mais qui avait été induite par la restructuration productive qui bouleversait le capitalisme à l'envers à travers du travail avec la marchandise information.

Vous avez mentionné la condition d'ancien combattant de la Résistance de mon directeur Desanti, très discret à ce sujet. J'avais eu vent de la légende, mais je ne l'ai connue en entier que lorsque Dominique Desanti, plusieurs années plus tard, a publié ses mémoires Ce que le siècle m'a dit. Et il a suggéré qu'une telle circonstance faisait partie de la scène que vous m'avez demandé d'évoquer. Je sais que les historiens de l'Occupation, mais surtout les historiens de la mémoire de ce traumatisme constitutif de la France contemporaine, ont commencé à voir dans 68 un tournant dans la vision de l'Occupation. Un tel revirement devrait être dans l'air du temps, je ne sais pas si les maoïstes de la Gauche Prolétarienne ont reniflé quelque chose à ce sujet, mais le fait est qu'en se présentant aussi comme les protagonistes d'une Nouvelle Résistance, il est tout à fait possible qu'en arrière-plan de cette absurdité complète se déroulait le nouveau fleuve de la mémoire et ses politiques de restauration et de vérité, à tel point que sans aucun préavis, en avril 71, est sorti le film décisif de Marcel Ophuls, Le chagrin et la pitié: chronique d'une ville française sous l'occupation, dans ce cas Clermont Ferrand. C'était le choc que l'on connaît. Seulement 10 ans plus tard le film est arrivé à la télévision. Je ne sais pas comment j'ai réussi à assister à une des premières séances dans une salle du Quartier Latin, le SaintSéverin. Deux ans après, un autre scandale, $L a$ France de Vichy, de Robert Paxton, dont j'ai suivi la controverse par la presse, mais ce livre je n'en suis venu à le lire que beaucoup plus tard. Pour être historiquement honnête, quand la boîte de Pandore des crimes de la Dictature a commencé à être ouverte au Brésil, mais surtout les misères de la « collaboration » locale, et que peu à peu, émergeait la perception horrifiée du consensus prédominant dans les années de plomb. A ce moment-là, le rôle de l'historiographie française du Présent a de nouveau été décisif. Précisément ce temps qui s'ouvre aux 
contemporains avec la "dernière catastrophe ", selon la formule d'Henry Rousso - pour nous sans doute le désastre de 1964, à la différence que l'Occupant a toujours été parmi nous. Depuis lors, et de plus en plus, l'histoire avec une lettre majuscule en est venue à être vécue comme une collection de malheurs à réparer et à prévoir, puisque le futur, à son image et sa ressemblance, ne pourrait annoncer rien d'autre que des menaces de répétition. Nous pouvons considérer si cette nouvelle relation entre la politique et le temps ne serait pas apparue à partir du manque de l'immédiat post-68.

Avec le critique littéraire Roberto Schwarz, vous avez été l'un des premiers lecteurs brésiliens de la dite École de Francfort. Cependant, une certaine lecture courante voit cette école comme étant trop eurocentriste et élitiste. Comment a été découverte et, d'une certaine façon, introduite, cette école de pensée dans un pays du tiers monde et périphérique comme le Brésil? En quoi peut-elle ou ne peut-elle pas être intéressante pour la lecture de cet objet qu'est le Brésil et de ce qui prolifere politiquement et culturellement dans ce pays? En outre, en quoi des auteurs comme Theodor W. Adorno, Herbert Marcuse ou Walter Benjamin demeurent ou non nos contemporains? Par exemple, qu'est-ce que serait penser le Brésil à travers le concept d'industrie culturelle?

P.A. - J'ai connu le critique littéraire Roberto Schwarz en 1965. C'était en fait le premier lecteur brésilien d'Adorno et Benjamin que j'ai rencontré. Il n'avait jamais entendu parler de cela, et quant à Adorno, je savais qu'il existait, n'ayant pas bien sûr la moindre idée de ce dont il traitait, en le voyant cité sur la scène de l'hôpital, au début de la Nuit d'Antonioni. Dans cette scène de vie ruinée en plein décollage de la modernisation italienne, une telle mention n'était nullement accessoire, et montre à quel point le débat italien était avancé à propos du scandale de la vie qui continue après la mort. Roberto n'était pas encore un lecteur systématique de l'école de Frankfurt. Par ailleurs, on ne savait alors rien de la réelle dimension de cette variante du Marxisme Occidental, sauf en Italie, comme je l'ai rappelé, et même en Allemagne, sa redécouverte en était encore à ses premiers pas. Ses références étaient pour ainsi dire en vrac. Il était enthousiasmé par le matérialisme hérétique de l'essai sur la Nouvelle Musique et je soupçonne qu'à cette époque, la Minima Moralia était déjà lue comme un manuel de savoir-vivre intellectuel, sans parler évidemment des Notes sur la Littérature qui commençaient à sortir. En cette même année où les frankfurtiens m'ont été présentés sans que je sache trop quoi faire avec eux, Roberto avait publié un article étonnant sur les $8 \frac{1}{2}$ de Fellini, totalement atypique même de la bonne critique de l'époque, un article dans lequel était déjà visible l'imprégnation intelligente, bien que discrète, du Benjamin de la Reproduction technique de l'œuvre d'art ${ }^{4}$. On voyait que c'était quelque chose de plus que de la bonne critique de cinéma, comme bientôt on percevrait que sa critique n'était plus seulement littéraire.

Il ne s'agit pas d'une simple transplantation, au contraire. D'abord, bien que puissante, c'était juste une autre référence, un autre modèle de pensée dans le cadre théorique de la gauche. Qui du reste ne tombait pas sur un territoire vierge, bien au contraire, déjà bien élaboré par la culture organisée sous forme d'études sociales et littéraires, dans une Faculté raisonnablement éclairée. Et aussi par le passage à travers un groupe mythique d'études du Capital, totalement en désaccord avec les conventions en vigueur dans les tendances marxistes classiques, et dans la façon dont ils interprétaient la condition périphérique particulière du Brésil, la marche ascendante des étapes de l'évolution économique et leurs tâches historiques respectives, comme l'inévitable révolution démocratique-bourgeoise, dans l'antichambre du socialisme, etc... L'adéquation toutefois était encore loin d'être pacifique. Notre précurseur et cicérone avait lu et bien lu Histoire et Conscience de Classe, considérée comme l'acte de naissance du Marxisme Occidental, et 
comme il restait fidèle au Lukács ultérieur, malgré tous les chagrins, il arrangea alors un mariage de convenance entre Lukács et Adorno : le premier pour les grandes questions du Réalisme, le second, pour les grands apories de l'Avant-Garde, auxquelles le Lukács de la normalisation soviétique était aveugle. Compte tenu que pour son professeur et maître, dont il était entre-temps devenu l'assistant, Antonio Candido, dont le nom à cette époque était déjà un programme pour le renouvellement des études littéraires - un programme de sondage en profondeur de l'expérience brésilienne à travers les formes de sa représentation littéraire, ni plus ni moins qu'un Auerbach révisé pour accueillir les révélations du premier, offert par une matière du second - Adorno et compagnie ont toujours été un livre fermé, rarement ouvert et presque toujours avec étrangeté. (Une génération plus tard, ces frankfurtiens de la première heure seraient encore écartés en tant que critiques en même temps sentimentales et abstruses d'un capitalisme qui n'avait pas encore dit son dernier mot dans la récupération des sociétés périphériques. Évidemment le royaume d'Antonio Candido n'a jamais été de ce monde, où se mélangent apologie et de non moins périlleuses questions de méthode).

J'insiste sur ce point, apparemment latéral, car nous sommes très proches du cœur de la matière. Après tout, vous voulez savoir combien de révélations et d'étrangetés a produit chez nous cette première version de la Théorie Critique, née de l'urgence de l'entre-deuxguerres européennes, et en se rendant peu à peu compte que l'implosion de la civilisation libérale-bourgeoise n'a pas seulement produit trois formations monstrueuses : le nazisme, le stalinisme et l'admirable nouveau monde américain sur le chemin d'une autre explosion (nucléaire cette fois). Mais aussi, plus étonnant encore, ils ont pris en compte que c'était aussi des formations au fond apparentées, parce qu'elles étaient nées du même ventre d'un système démentiel de transformation du travail en argent comme une fin en soi. Une révélation sans doute choquante. Car tout l'effort de guerre, orienté vers la défaite militaire du nazisme à travers une alliance in extremis entre les sociétés de marché de l'Atlantique Nord et le socialisme bureaucratique de l'Union Soviétique, a été alimenté par la croyance sans doute crédible qu'il s'agissait d'une question de de vie ou de mort entre les héritiers des Lumières et les représentants d'une impensable régression barbare. Les Grands Espoirs de 1945 dans le renouveau paradoxal d'une civilisation qui s'était auto-détruite jaillirent justement de cette transposition des fronts de guerre en une victoire lumineuse de la coalition des lumières entre les libéraux et les communistes sur les forces exterminatrices des ténèbres. Pour en revenir au commentaire de la question précédente : pour mesurer le grand faisceau d'attentes qui rayonnait depuis l'Année Zéro de 1945, il suffit de rappeler, toujours dans l'exemplaire drame français, que toute la période de la Résistance avait été vécue comme l'antichambre de la révolution, avec la libération du joug nazi en Europe, "les lendemains qui chantent", annoncés par Gabriel Péri au moment d'être fusillé, étaient finalement à portée de main, et il était simplement inconcevable qu'après une telle bataille dans les ténèbres, la vie continue comme avant. Mais c'est ce qui a eu lieu, en 1947 il n'y avait plus d'illusions à son sujet, la Collaboration n'avait pas seulement compensé comme au fond avait vaincu, et le «trésor des révolutions » (Hannah Arendt) avait été enterré à nouveau. Adorno et Horkheimer n'ont pas attendu la fin de la guerre pour se rendre compte de l'énorme point aveugle de ce triomphe de l'Aufklärung: que le processus des lumières était à la rigueur un processus de domination (en commençant par la Nature), commun aux trois idéologies : libéralisme, socialisme et fascisme. Ils ne s'attendaient pas non plus à ce que la Guerre Froide commence (encore une fois sur deux fronts, l'un géopolitique et l'autre idéologique) pour 
montrer que des deux côtés de l'infâme rideau de fer prospéraient des sociétés similaires et rassemblées autour de la logique de l'administration totale.

Sans aucun doute cette vision était eurocentriste, mais là aussi il y avait un eurocentrisme d'un genre nouveau et contre-intuitif, car si c'est un fait qu'ils ignoraient et ne prenaient pas en compte la dynamique spécifique de l'ancienne frange coloniale du système, après le Tiers Monde (ni même Marcuse de l'activisme anti-guerre du Vietnam), ils ne se sont jamais trompés à propos du consensus keynésien qui a présidé la reconstruction européenne, le mirage des "trente glorieuses » d'un capitalisme organisé et donc libéré des crises, une sorte de machine de croissance auto-régulée. Car dans le Brésil de 45 , le développementisme qui était alors à l'aube de son développement se reflétait précisément dans ce modèle européen du capitalisme normalisé. Et même l'horizon cepalien $^{5}$ de dépassement du sous-développement, même s'il s'opposait à l'étapisme des politiques de modernisation préconisées par le centre hégémonique de l'époque et comportait ainsi dans une certaine mesure une dose de négation et critique, empêchait même la compréhension que le paradigme qui guidait notre recherche d'une sortie nationale pourrait être empoisonné. Et il en a ainsi été de même avec le marxisme hétérodoxe, celui-là même du Séminaire Marx, qui, selon Robert Schwarz, aurait engendré une "nouvelle intuition" du Brésil, étant cependant, au mieux, un marxisme industrialisant, selon la formule avec laquelle il clôt sa récapitulation du parcours intellectuel qui a conduit l'un des membres de ce groupe à la Présidence de la République.

Dans ces conditions impératives de manœuvrer le capitalisme en faveur d'une construction nationale, comment pouvons-nous espérer qu'une lecture, même éclairée, des essais d'Adorno sur la critique culturelle (sans parler du diagnostic de l'époque) pourrait parler à l'imagination intellectuelle et politique d'un Antonio Candido (pour rester avec le plus grand de tous), à l'exception des affinités significatives de la méthode critique, comme Roberto l'a souligné beaucoup plus tard? C'est pour cela, que ce qui me frappe le plus quand je me souviens de l'enthousiasme de notre critique vis-à-vis de l'essai d'Adorno, est la manière dont ce flagrant horizon rabaissé pouvait cohabiter avec les grandes attentes du moment, dont le temps était l'ascendant et pointait vers une rupture, disons-le de manière classique, aussi intensément, vers ces attentes, que même le coup définitif de 64 n'avait pas refroidies - il suffisait de déboucher la cocotte-minute comme nous l'avons rappelé tout à l'heure.

19 Laissons l'exception de Marcuse de côté, je ne peux que mentionner en passant l'énorme désaccord de 68 entre la Théorie Critique et l'explosion de Mai : alors que la nouvelle gauche dans les rues et les occupations se reconnaissait dans le Grand Refus, curieusement ressuscitée dans un cercle de mandarins, Adorno et compagnie ne cachaient pas leurs perplexités devant des cocktails Molotov jetés en raison du modèle de pensée dont le ressort secret venait de la constatation que le devenir monde de la philosophie, dans la prophétie des classiques qui justement concevaient le monde comme une crise, n'avait pas été accompli et avait quitté la scène. Avant d'en revenir au Brésil, je ne peux pas m'empêcher de rappeler - puisque vous m'avez posé une question sur l'actualité de la Théorie Critique originale - que la théorie marcusienne de la société unidimensionnelle disait exactement cela : que le capitalisme dit organisé par l'État était précisément cette cocotte à exploser, par la démesure de la répression dans toutes les sphères de la vie, fonctionnellement superflue à l'époque d'un énorme développement des forces productives qui, cependant, ne pouvait plus distinguer son envers destructeur: la symbiose entre l'affluence et le Warfare. Il va sans dire que rien de tout cela ne pouvait 
faire sens pour une gauche nationale, aussi déconcertée par le développement du sousdéveloppement, comme Gunder Franck le disait ici. Mais n'était-ce pas ainsi la « marge » antagoniste imaginée par un Marcuse mécréant de la capacité disruptive de la critique sociale immanente au cœur du système?

Mais avec cela je suis déjà de retour sur la piste brésilienne, en fait double, au bord de la duplication de la personnalité intellectuelle, dans le cas du critique Roberto Schwarz, au moins dans ce bloc le plus agité des années 60 : Marxisme Occidental d'un côté - comme il a autrefois été interprété, rien de moins qu'une théorie critique précisément d'une défaite historique, celle de la Révolution, au moment le plus fort de la crise du XXe siècle - d'autre part, au minimum compagnon de route de quelque chose de proche d'un socialisme national-populaire, comme notre époque l'exigeait et nous nous laissions tous porter par ce courant. Je n'ai pas perdu le fil: savoir si oui ou non la Théorie Critique a été décisive ou non dans le renouvellement de la perception du pays hérité de la meilleure tradition critique brésilienne. Une autre singularité nationale : cette tradition, dont la première cristallisation remonte à une méditation agonisante de plus d'un siècle à propos de notre anomalie de naissance, la fusion du capitalisme et de l'esclavage. Car je pense qu'une telle fécondation mutuelle s'est produite, et peut être reconnue, dans le cycle machadien de Roberto Schwarz. Je pense même, sans aucune exagération, que dans ce cycle d'essais aujourd'hui classiques, ceux qui cherchent trouveront plus que la graine de tout un programme d'étude et d'intervention, non seulement pour la critique littéraire brésilienne, mais pour une éventuelle renaissance de la tradition critique à laquelle nous nous référons, dont l'origine radicale remonte à Roberto qui l'a redécouverte précisément dans le Machado de Assis de la maturité. Je rappelle que, selon Roberto, dans la prose fictive du second Machado, le narrateur est toujours un type social dont le goût raffiné, régi par la norme bourgeoise de l'apogée libérale, coexiste, sans plus grands états d'âme, avec le substrat barbare local. L'effet satirique est double, et la démoralisation des deux pôles idéologiques en confrontation, favorisée par le surréaliste esclavagisme libéral qui nous a défini.

21 Par conséquent je pense que Roberto Schwarz a fait un pas en avant qui n'aurait pas été possible sans l'imprégnation réfléchie et productive de la Théorie Critique originale, quelque chose comme un jugement sur le temps présent à partir de la note spécifique de la matière brésilienne. Mais pas seulement, jusque-là ça a été Antonio Candido. Voici le pas en avant: ce temps présent n'est pas une coupure quelconque, mais une époque, la nôtre, dont la date inaugurale est une Chute, un désastre sans retour, après lequel, cependant, la vie et sa reproduction sociale continuent, mais dans un mode endommagé sans rémission. Comme je l'ai rappelé dans la réponse précédente, l'historien Henry Rousso a écrit un livre pour montrer que non seulement c'est le cas - tout Temps Présent, plus précisément, toute histoire dite contemporaine, commence par une catastrophe, la dernière - mais en constatant, de plus, que le phénomène par excellence que scande le temps historique occidental moderne, depuis la Révolution Française, est toujours la sortie d'une guerre, et une guerre pour ainsi dire cataclysmique, de sorte que l'intérêt pour le passé proche semble inéluctablement lié à un moment de violence paroxystique. Et non plus une violence quelconque. Et en cela la périphérie capitaliste est un laboratoire privilégié, dans un contexte de machinerie de l'accumulation primitive et de la chasse à l'homme qui lui est corrélée. Il n'est pas difficile de nous en rendre compte avec notre dernière catastrophe, et pas non plus de comprendre pourquoi, pour les Français, juin 1940 pourrait être le début du Présent, du reste confirmé par la brèche historique qui 
s'est refermée, paradoxalement, lors de la sortie victorieuse de la guerre. Notre dernière catastrophe a été les 20 ans de dictature, comme je l'ai dit. Car ça été la révélation que, à partir du Coup d'Etat, l'élite brésilienne était prête à tout dans l'échelle de l'horreur, et, celle latino-américaine, durant les 30 années de la Terreur Blanche qui a suivi, et qui a fourni à notre critique la nouvelle optique par le prisme lequel il a finalement reconnu l'actualité de Machado de Assis. Et cela lui a permis d'en tirer les conséquences que l'on sait. Ou plutôt, qu'on ne sait pas autant que ça. Par exemple, que le temps mort des romans machadiens était de retour avec le grand trauma de la naissance du Brésil contemporain en 1964. Et ce n'est pas tout. Le fait de reconnaître qu'avec l'interdiction subséquente de l'attente déjà quelque peu imaginée de surmonter le sous-développement était encore plus important et décisif, ce n'est pas pour cela que la vie cesserait de se reproduire avec les caractéristiques sociales propres à une rétrogradation sans précédent et qui avait, bien sûr, une correspondance dans le centre organique du système, qui a également commencé à se périphériser avec l'implosion continue de la société de travail qu'avait engendrée le compromis de la post-guerre. En adoptant le diagnostic des théoriciens allemands de la Critique de la Valeur, dès le début des années 1990, Roberto a commencé à reconnaître dans notre « société vaincue » une société post-catastrophe ${ }^{6}$.

Je ne vais pas, bien sûr, énumérer les symptômes, il suffit de regarder autour de soi, car je pense que, il y a une génération, cet état d'urgence s'étendait de la périphérie vers le centre. Je voudrais souligner que le point névralgique de ce schéma réside dans la redéfinition de la reproduction sociale après la Chute, qu'une fois Roberto lui-même, en plein chapitre Collor de la crapulisation de l'élite brésilienne, a décrite comme la disposition absurde de continuer à mener sa vie dans des circonstances de plus en plus impossibles. Ni plus ni moins, c'est le jugement irrévocable d'Adorno, depuis les premiers temps de la sortie de la Grande Guerre de 30 ans terminée en 45, et dont la logique de désintégration a commencé à être enregistrée dans les Minima Moralia. Une des métaphores de notre temps est la fondamentale Chute, que Roberto a formulée, en relisant le grand essai d'Adorno sur le Beckett de Fin de partie, une fin qui « arrive » dans une Europe post-bombe et champ, de nombreuses années après avoir atteint la clé de la dialectique tronquée dans les romans de Machado. Sans les vases communicants qui le reliaient plus à l'esprit qu'à la lettre d'un Adorno, il ne pourrait pas profiter de la constatation décisive que la dialectique qui surmonte des classiques ne fonctionne pas au Brésil. Pas aussi simple que ça, mais je suis allé trop loin, au-delà bien sûr de cette énormité qui a été de penser le Brésil pour ainsi dire « après la fin du monde » (comme il est possible de le lire dans le titre d'un livre de Marildo Menegat sur Adorno), plus précisément, après notre dernière catastrophe, reste à savoir laquelle, en passant le curseur sur la ligne du temps brésilienne maintenant que nous le voyons se dérouler dans le lit même du temps du monde qui à soixante-dix ans, se mesure symboliquement par la plus ou moins grande proximité de sa propre fin.

Je m'explique: depuis 1947, la Société des Scientistes Atomiques, fondée par des dissidents du projet Manhattan, publie un bulletin annuel dans lequel apparaît le diagramme d'une horloge, dont les aiguilles ou s'approchent ou s'écartent d'une non équivoque heure finale, minuit, dans laquelle s'associent les dernières mythiques ténèbres à une explosion cataclysmique scientifique manufacturée. Ces dernières années, dans l'éditorial explicatif, des données ont été ajoutées sur les progrès de la bombe climatique et de ses dérivés. Cette figuration particulière du nouvel état de choses - la vie qui continue après que l'impensable a eu lieu - vaut ce que valent certainement tous les autres emblèmes de 
l'époque, mais tout le monde a compris quand on a avancé l'Horloge du Jugement Dernier de 3 à $2 \frac{1}{2}$ minutes, une semaine après l'inauguration de Donald Trump. Quand je dis que tout le monde c'est même tout le monde, pas seulement ceux qui ont l'habitude de prédire l'apocalypse, avec les frankfurtiens en haut de la liste, mais maintenant les intégrés, l'establishement global en état de choc: rien que l'année dernière, avec des événements extrêmes comme le Brexit et Trump, sans parler de la récente avalanche migratoire, certainement le phénomène disruptif par excellence de cette dernière décennie, sans oublier la vaste et insolite insurrection électorale de la droite populiste, et l'arrière-plan du terrorisme comme toujours. Ce sont maintenant les intégrés qui parlent d'une Grande Régression, que je mentionne seulement pour indiquer combien l'autre côté, pour ainsi dire, a commencé à raisonner politiquement en termes d'attentes déclinantes, car après tout, c'était une autre sortie de guerre qui en frustrant a marqué un autre présent, je me réfère aux Grandes Attentes nourries par le côté gagnant de la Guerre Froide. Ça a été presque 30 ans de refoulement. Cela dit, je ne sais pas si je réponds complètement à votre question sur l'actualité de la Théorie Critique originelle, dont l'inactualité est habituellement mise en évidence parce qu'elle a lié sa fortune critique à la survie indéfinie de l'arrangement fordiste de l'immédiat après-guerre, et comme cet ordre a cessé d'exister avec la victoire du contrecoup néolibéral, elle serait au moins restée sans sujet. Comme ils ne se sont jamais laissé convaincre par la façade pacifiée de ces sociétés après la catastrophe... Nous y entrons.

\section{NOTES}

1.

Tentative d'identification de l'idéologie française

2.

Note du traducteur : Tous les mots et expressions en français et en italique dans le texte sont aussi présents dans le texte d'origine.

3.

Ironie supplémentaire: durant une bonne partie des années 1960 et autour des années 80 pour une autre saison dans le Département, dont il a contribué à compléter la formation, Gérard Lebrun a été notre dernier maître à penser. J'ai presque été l'un de ses élèves, bien que je n'aie assisté qu'à une partie de certains de ses cours mémorables.

4.

Dans la traduction française le texte a été publié sur le titre: L'œuvre d'art à l'époque de sa reproductibilité technique.

5.

Note du traducteur: Paulo Arantes fait ici référence à la CEPAL (Commission économique pour l'Amérique latine et les Caraïbes) crée par l'ONU en 1948.

6. 
L'expression "société vaincue » se trouve dans Ornitorrinco de Francisco de Oliveira, un essai dans lequel il fait une évaluation globale des conséquences de l'effondrement de la modernisation brésilienne, désactivé à la suite de la Troisième Révolution Industrielle et de ses conditions prohibitives. Faisons le calcul. D'abord vaincue par la violence politique du Coup d'Etat de 64. Vaincue en sortant de la Dictature comme une société de désindustrialisation endettée. Et vaincue maintenant par un second coup d'Etat comme une farce. Tout ensemble, trois fois un « désistement historique » - toujours à l'initiative d'une classe dirigeante se reproduisant aujourd'hui décidément sous le signe de la délinquance - le long desquelles nous nous sommes transformés dans le monstre social d'aujourd'hui. Dans la préface en 2003 du livre de Chico de Oliveira, je ne sais pas s'il pensait davantage développer plus tard l'argument, Roberto Schwarz a fourni la règle et le compas de notre, à savoir : que l'anti-climax lié à l'épuisement du développementisme, épuisé sans réaliser ce qu'il promettait, en se faisant l'écho d'un autre et se révéla être le cousin d'un autre Big Bang, la frustration des moments d'espoir inhabituel éveillés par la victoire de 1945 sur le nazi-fascisme. Cela a coûté, mais finalement est arrivé, en 1964, le message que les portes qui se sont ouvertes en 1945 pour une forme supérieure de société étaient fermées depuis toujours, au moins par des voies systémiques. Jusqu'à ce que l'effondrement du monde soviétique tire la voie anti-systémique faussement alternative. Et attention: une fois dans une préface à une édition du 18 Brumaire, et en corrigeant Marx, Marcuse alertait sur la réelle tragédie de la répétition comme farce de l'histoire, qui aurait ouvert depuis lors la voie au fascisme dès lors que la société capitaliste ne peut plus se gouverner sans faire appel à la politique de la terre brûlée du lumpensinat en service. La nôtre a toujours été une lumpen-bourgoisie, comme s'il y en avait une autre, l'éternel rêve de consommation du progressisme national.

INDEX

Mots-clés : Theodor W. Adorno, Roberto Schwarz, Ecole de Francfort, périphérie, dictature militaire brésilienne, catastrophe

\section{AUTEUR}

\section{PAULO ARANTES EN ENTRETIEN AVEC FRED LYRA}

Philosophe, Sao Paulo et doctorant à l'Université de Lille 